\title{
An empirical study on hospital-based prevention of recurrent urinary stone disease in Germany
}

\author{
Alina Reicherz ${ }^{1} \mathbb{D} \cdot$ Patricia Rausch $^{1} \cdot$ Roman Herout $^{2} \cdot$ Joachim Noldus $^{1} \cdot$ Peter Bach $^{1}$
}

Received: 22 April 2021 / Accepted: 11 August 2021 / Published online: 18 August 2021

(c) The Author(s) 2021

\begin{abstract}
Purpose Urinary stone disease is a common disease with a prevalence of $4.7 \%$ in Germany. The incidence increased over the last decades, and recurrence rates are up to $50 \%$ in the first 5 years after diagnosis. Adequate preventive measures can avoid up to $46 \%$ of stone recurrences. These numbers outline the importance of prevention. Especially among high-risk stone formers, specific diagnostics and measures are required. Published data indicate the divergence between the importance of prevention and its implementation in everyday clinical practice. This is the first survey among German urological departments highlighting medical care concerning the prevention of recurrent urinary stone disease, identifying challenges and providing recommendations for improvements.

Methods Two hundred and seventy urological hospital departments in Germany were anonymously surveyed about measurements to prevent recurrent stone disease. The questionnaire comprised 23 items dealing with diagnostics, counselling, knowledge among doctors concerning preventive measures and difficulties in preventing recurrent urinary stone disease.

Results Sixty-three urological departments (23.8\%) answered the survey. The majority perform stone analysis at first and repeat events. Most patients with urinary stone disease receive general advice on preventive measures during their hospitalization. General recommendations focus on fluid intake and lifestyle changes. However, specific diets are infrequently recommended by inpatient urologists. Diagnostics to identify high-risk stone formers are mostly insufficient, and guidelinecompliant urine tests are uncommon.

Conclusion The quality of secondary prevention needs to improve considerably. The focus should be put on identifying high-risk stone formers and offering those patients specific counselling. Furthermore, general advice on dietary recommendations should be extended.
\end{abstract}

Keywords Prevention $\cdot$ Recurrent urinary stone disease $\cdot$ Survey $\cdot$ Empirical study $\cdot$ Germany

\section{Introduction}

Urolithiasis is a common disease, $12 \%$ of the world's population suffers from urolithiasis once in a lifetime [1]. Over the last few decades, an increase in the incidence and prevalence rates was observed [2]. A continuous upward trend is expected due to a change in nutrition, lifestyle and rising temperatures caused by climate change [3]. The recurrence

Alina Reicherz

alina.reicherz@rub.de

1 Department of Urology, Marien Hospital Herne, RuhrUniversity of Bochum, Hölkeskampring 40, 44625 Herne, Germany

2 Department of Urology, University Carl Gustav Carus, Dresden, Germany rate in stone patients is up to $50 \%$ within the first 5 years [4, 5]. Depending on the stone composition and causal disease, the recurrence rate can increase to $100 \%$ if no preventive measures are taken. The recurrence rate has increased twofold between 1979 and 2000 in Germany [5]. The observed and expected increase in patients with urinary calculi outlines the exigency to take action.

Nolde et al. showed that preventive measures can reduce relapse in up to $46 \%$ of stone patients [6]. Most stone patients require basic preventive measures comprising recommendations on fluid intake, diet and lifestyle changes. The German and European guidelines define groups at low and high risk for stone recurrence. About one quarter of patients has a high-risk profile. For high-risk stone formers, however, specific measures need to be taken to prevent a recurrence. 
Recommendations are mainly based on experts' opinions, as prospective randomized trials are rare [7]. In Germany, medical care concerning secondary prevention of urinary stones is mainly provided by specialized counselling hours in hospitals and outpatient clinics. However, it is not known to what extent it is implemented in daily routine. Prevention of urinary stone disease is poorly documented in the literature. A Canadian retrospective study on recurrent nephrolithiasis, published by Krepinsky et al. (2000), showed that in only $35.1 \%$ of patients presented for a metabolic investigation, a complete, guideline-compliant evaluation was performed [8]. A model calculation from 2006 showed that adequate diagnostics and prevention could save the German health care system about 178 million Euros per year [9].

This German-wide survey aims to analyze the current state of medical care regarding the prevention of stones, outline major challenges and provide recommendations to improve medical care.

\section{Methods}

This survey is a German-wide empirical analysis evaluating the state of medical care concerning recurrent stone disease prevention. We designed a non-validated, anonymous questionnaire and sent it by email to directors of all German urology departments $(n=270)$ in February and March 2021. In the email, we asked for one physician to complete the questionnaire representatively. The questionnaire was designed using REDCap ${ }^{\circledR}$. It comprised 23 questions dealing with metabolic evaluation, counselling of patients, and physicians' knowledge on preventive measures. We used Likert scale questions and multiple-choice questions. The answers matched recommendations of the German guidelines on urolithiasis [7].

Finally, we calculated descriptive statistics, including mean, standard deviation of the mean (SD), and interquartile range (IQR) for variables using Excel $^{\circledR}$, Version 2103.

\section{Results}

In total, 63 of 270 urological departments responded to the survey and were included in the analysis. Five emails could not be delivered, resulting in an overall response rate of $23.8 \%$.

Urological departments answering the questionnaire treat a total of 35,445 patients with urinary stone disease per year (cases estimated by responders). The mean number of patients treated with urinary stones per hospital is 572 (SD 358; IQR 300-700; estimation by responders). $28.6 \%$ of the responders were from academic hospitals.

\section{Stone analysis}

The majority of the urological departments (50.8\%) always analyze the stone's composition in first-time stone formers; $22.2 \%$ responded that they mostly analyze it, $7.9 \%$ occasionally analyze it, $15.9 \%$ rarely analyze it, and $3.2 \%$ never analyze it. Repeat stone analysis is always performed in $49.2 \% .11 .5 \%$ answered they repeat stone analysis if recurrence occurs under pharmacological treatment, $21.3 \%$ repeat it if recurrence is shortly after interventional therapy with complete stone removal and $27.9 \%$ if relapse occurs after a prolonged stone-free period, while $8.2 \%$ never perform repeated stone analysis.

\section{Diagnostics}

In patients with a high risk of recurrence, the majority of treating urologists $(38.1 \%)$ rarely request repeated urinary pH measurements, while $9.5 \%$ never do so, $14.3 \%$ occasionally do, $17.5 \%$ mostly do, and $20.6 \%$ always include them in their diagnostic pathway.

For patients at high risk, 24-h urine collection is less often included in the standard diagnostic repertoire than repeat urinary $\mathrm{pH}$ measurements: $22.2 \%$ never, $30.2 \%$ rarely, $17.5 \%$ occasionally, $19.0 \%$ mostly, and $11.1 \%$ always perform a 24-h urine collection in patients with a high risk for recurrence.

Urologic departments were asked which blood test they run for patients with stones containing calcium oxalate or calcium phosphate. Results showed that blood laboratory analysis includes serum electrolytes in $93.5 \%$, creatinine in $93.5 \%$, uric acid in $77.4 \%$, urea nitrogen in $82.3 \%$, glomerular filtration rate in $80.6 \%$ and parathyroid hormone in $56.5 \% .4 .8 \%$ do not evaluate any of the parameters.

Hounsfield units indicating the stone's density can be included in the decision-making process for stone treatment. The handling differs between urologic departments: $14.3 \%$ never consider Hounsfield units, while $20.6 \%$ rarely do, $25.4 \%$ occasionally do, $22.2 \%$ mostly do, and $17.5 \%$ always do.

\section{Counselling}

Urologic departments were asked if counselling for preventing recurrent urinary stone disease was mainly the outpatient clinic's responsibility. $11.5 \%$ agreed that it is the duty of urologists in outpatient clinics; the majority (44.3\%) mostly agreed, while $24.6 \%$ partially agreed and $19.7 \%$ disagreed.

In $54.1 \%$ of urological departments, urologists mostly and in $34.4 \%$ always give general advice during rounding or discussion upon discharge. Most departments (65.6\%) do not 
offer an additional, specialized consultation hour for stone formers, while $19.7 \%$ offer an additional consultation hour for selected patients. In 14.8\%, additional consultation hours are a regular part of medical care. Those clinics counsel on average about 57 patients per year [IQR: 20-50].

Urologists were asked to specify the content of counselling. General advice on intake of fluids comprises the amount (98.4\%), circadian drinking (73.8\%) and type of beverage (77.0\%). Regarding nutrition, urologists mention a balanced diet (93.4\%), protein intake (47.5\%), salt intake (44.3\%), oxalate intake (62.3\%) and calcium intake (57.4\%). Almost always (95.1\%) lifestyle including activity, normalizing body weight and reducing stress are discussed.

Within counselling hours, the advice on fluid intake and nutrition is more comprehensive: $100 \%$ give recommendations on the amount, $81.0 \%$ on circadian drinking and $95.2 \%$ on the beverage type. Concerning nutrition, urologists discuss a balanced diet $(90.5 \%)$, protein intake $(81.0 \%)$, salt intake $(76.2 \%)$, oxalate intake $(85.7 \%)$ and calcium intake $(85.7 \%)$. Commonly (95.2\%), urologists advise on lifestyle.

Professional nutritional counselling is not standard of care considering that $4.9 \%$ answered they mostly offer, $27.9 \%$ occasionally offer, $37.7 \%$ rarely offer, and $29.5 \%$ never offer nutritional advice.

To assess the quality of preventive measures in German urological departments, we asked urologists if they agreed with the statement "counselling for patients with recurrent stone disease is excellent in our department". Answers point out that urologists question their work: $6.6 \%$ agreed with the statement, the majority (47.5\%) agreed partially, $27.9 \%$ agreed predominantly, and $18.0 \%$ disagreed.

\section{Risk factor}

Urologists were asked if they think that the attention drawn to risk factors like stone composition, family history, metabolic diseases and anatomic abnormalities is sufficient. The majority (49.2\%) thought that the attention on these risk factors is partially sufficient, and $29.5 \%$ stated that it is predominantly sufficient (Fig. 1).

We tried to identify risk factors that are of little concern despite their importance. $77 \%$ agreed that more attention should be put on early occurrence (children or teenagers). Also, more attention should be turned on positive family history, stones containing urine acid, infectious stones and solitary kidneys (Table 1).

We aimed to identify associated diseases that are not given enough consideration (Table 1). $70.5 \%$ of urologists answered that more attention should be paid to hyperparathyroidism in future. Responders also see an increased need for awareness about metabolic syndrome, nephrocalcinosis, chronic gastroenteritis and bariatric surgery, primary hyperoxaluria and renal tubular acidosis.

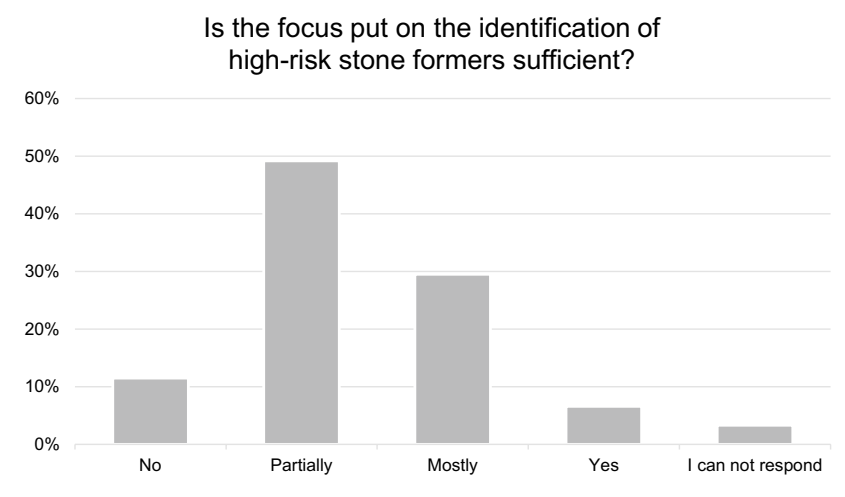

Fig. 1 Identification of high-risk stone formers. $n=61$, Likert scale (only one response possible)

\section{Success rates of secondary prevention}

We asked responders to estimate how many recurrent stone episodes can be prevented by good counselling on preventive measures. On average, urologists answered $36.2 \%$ (IQR $20-50$ ) with a widespread range of $10-90 \%$.

\section{Knowledge}

The survey included two questions on the knowledge of preventive measures. Responders were asked to self-assess their knowledge and their co-workers' knowledge in school grades (1-6; 1 corresponds to very good, and 6 to inadequate). On average, urologists rated themselves 2.6 (IQR 2-3) and their colleagues 2.9 (IQR 2-3).

\section{Additional comments}

Responders remarked on the time intensity and insufficient reimbursement of diagnostics and stated that these factors cause neglect and result in inadequate training. Responders repeatedly commented on poor patients compliance concerning preventive measures.

\section{Discussion}

Adequate diagnostic algorithms and preventive measures can reduce recurrent stone disease by $46 \%$ [6]. Per year 200,000 patients are estimated to have recurrent stone episodes in Germany [5]. Hypothetical that equals 80.000 patients experiencing avoidable stone recurrence. Nephrolithiasis is associated with an increased risk for chronic kidney disease and hypertension $[10,11]$. Therefore, prevention is critical to prevent both an impact on quality of life and comorbidities. Moreover, it also has tremendous economic benefits; treatment costs could be lowered, and the loss of productive work 
Table 1 Risk factors and associated diseases that are of little concern despite their importance, according to hospital-based urologists

\begin{tabular}{ll}
\hline Multiple choice question & \\
\hline Risk factors that are of little concern despite their importance & \\
Early occurrence (in children or teenagers) & $77.0 \%(47 / 61)$ \\
Positive family history & $63.9 \%(39 / 61)$ \\
Brushite containing stones & $31.1 \%(19 / 61)$ \\
Urine acid stones & $54.1 \%(33 / 61)$ \\
Infectious stones & $50.8 \%(31 / 61)$ \\
Single kidneys & $47.5 \%(29 / 61)$ \\
None & $3.3 \%(2 / 61)$ \\
Associated diseases that are not given enough consideration & \\
Hyperparathyroidism & $70.5 \%(43 / 61)$ \\
Metabolic syndrome & $63.9 \%(39 / 61)$ \\
Nephrocalcinosis & $32.8 \%(20 / 61)$ \\
Polycystic kidney disease & $16.4 \%(20 / 61)$ \\
Chronic gastroenteritis and bariatric surgery & $67.2 \%(41 / 61)$ \\
Sarcoidosis & $18.0 \%(11 / 61)$ \\
Neurogenic bladder dysfunction caused by spinal cord injuries & $29.5 \%(18 / 61)$ \\
Cystinuria & $36.1 \%(22 / 61)$ \\
Primary hyperoxaluria & $44.3 \%(27 / 61)$ \\
Renal tubular acidosis & $54.1 \%(33 / 61)$ \\
2,8-dihydroxyadeninuria & $19.7 \%(12 / 61)$ \\
Xanthinuria & $31.1 \%(19 / 61)$ \\
Lesch-Nyhan syndrome & $16.4 \%(10 / 61)$ \\
Cystic fibrosis & $21.3 \%(13 / 61)$ \\
None & $3.3 \%(2 / 61)$ \\
\hline
\end{tabular}

time could be reduced [12]. Despite these facts, this survey outlined that diagnostics and patient counselling for urinary stone formers in Germany have room for improvement. To meet patients' needs, urologists should tap the full potential of secondary prevention.

\section{Area of responsibility}

To our knowledge, so far, no data existed on the extent of secondary prevention of urinary stones in German in- and outpatient clinics. Our survey showed that almost $90 \%$ (54/63) of urologic departments mostly or always give general advice during rounding or final discussion. Counselling for high-risk stone formers requires follow-up appointments for a specific metabolic workup. It is not reasonable during an acute stone event, as patients should be stone-free for at least 20 days before evaluation. Urologists working in hospitals regard counselling to prevent recurrent stone disease as the outpatient clinic's area of responsibility, and most departments do not offer an additional specialized consultation hour; academic hospitals offer it more often $(33.3 \%$ vs. $6.7 \%)$. In our view, medical care would benefit from a discussion about who is to hold responsible for preventive measures. A first approach could be an adequate reimbursement. This is neither given in an outpatient nor in an inpatient clinic.

\section{General advice}

Responders' subjective grading of preventive measures in their urological department outlines that the quality is heterogeneous. While general advice on fluid intake and lifestyle, including activity, normalizing body weight and reducing stress is often sufficient, advice on a diet is incomplete. Protein, salt, oxalate and calcium intake are discussed in approximately half of the urologic departments during rounds. Patel et al. questioned members of the North Central Section of the American Urological Association and the Endourological Society on medical management of urolithiasis [13]. In comparison, they showed that $47 \%$ versus $69 \%$ recommend a low animal protein intake to all stone formers, while $3 \%$ versus $4 \%$ give recommendations only to calcium stone formers, $39 \%$ and $22 \%$ only to uric acid stone formers, and $10 \%$ versus $5 \%$ do not counsel their patients about low animal protein intake. One can speculate that different health care systems, especially legal and pecuniary differences, contribute to the difference. This is also evident from the "Additional Comments" of the survey mentioned in "Results". 


\section{High-risk stone formers}

Stone analysis is the basis for further diagnostics, treatment plans and identification of high-risk stone formers [14]. The majority (46/63) of urological departments always or mostly analyze urinary stones at the first event. However, about $19 \%$ of the responders do not follow the guidelines' recommendations. They stated that their department never or rarely analyzed urinary stones. To detect a change in the composition, the majority perform stone analysis in repeated stone events.

General advice concerning preventive measures is recommended by German, European and American guidelines, while an extended diagnostic algorithm based on the composition of the stone is required for stone formers at high risk to rule out associated metabolic diseases [7, 14, 15]. In 2014 an American retrospective study evaluated the prevalence of 24-h urine testing among patients at high risk for recurrence; it was $7.4 \%$ among 28,836 patients identified [16]. Our survey shows that in Germany, repeated urine $\mathrm{pH}$ measurements and 24-h urine collection are also not routinely requested.

Necessary blood tests for patients with stones containing calcium oxalate or calcium phosphate are mostly run according to the guidelines. However, about $45 \%$ do not evaluate parathyroid hormone, and more than $20 \%$ do not evaluate uric acid.

About $25 \%$ of the patients who form stones are at high risk for recurrence [17]. Counselling should be offered to those patients. This survey outlines the challenges we face; urological departments counsel only $3.9 \%$ (estimation by responders) of all patients they treat with urinary stone disease. This equals an estimated one in six high-risk patients who undergoes an extended diagnostic algorithm.

Results of the survey demonstrated urologists' lack of awareness for risk factors (Fig. 1). Only about one-quarter of the urologists thought that the attention drawn to risk factors like stone composition, family history, metabolic diseases and anatomic abnormalities are predominantly or entirely sufficient. Responders agreed that there is a wide range of risk factors and associated diseases of little concern despite their importance (Table 1). The included self-assessment results show that urologists are only "satisfied" with their knowledge and their colleagues' knowledge on preventive measures (in German school grades $3+$ to 3 , corresponds to $\mathrm{c}$ in the American grading). This finding is not surprising. In a survey by Wertheim et al. from 2013 on nutrition in the management of urinary stones, members of the Endourological Society were questioned about providing nutrition recommendations. $52 \%$ of endourologists reported having low confidence in providing specific strategies to achieve optimal calcium intake [18].

Our results show that professional nutritional counselling is not standard of care in Germany. However, to improve counselling, nutritionists could be further included in medical care. This would bridge gaps of knowledge and lack of time in daily hospital practice. A survey distributed to American registered dieticians revealed that few stone formers are provided with nutritional counselling. About $50 \%$ of dieticians provide medical, nutritional counselling for urolithiasis, but $80 \%$ of them estimated that they see only one to two patients per month [19].

Reasons for neglect of secondary prevention of urinary stone disease should be discussed. Among improved techniques of endourological stone removal, a lack of time and low financial incentives, a wrong understanding of the patients' demands might be potential reasons. A survey among 159 stone formers and endourologists regarding the demand found that "most patients with stones will consider preventive medical therapy to avoid recurrent pain or a surgical procedure. In contrast, most urologists perceive that patients prefer to avoid medication even if it means tolerating several acute stone events and/or surgical procedures" [20].

Our study has the following limitations. First, the questionnaire is non-validated. This limits the reliability and validity of findings. A second limitation is that because the response rate is low it might be underpowered and pose a potential bias. However, in comparison, the survey on the medical management of urolithiasis among members of the North Central Section of the American Urological Association and the Endourological Society achieved a response rate of $14.4 \%$ and $9.1 \%$, respectively [13].

Our study provides relevant data as there is no record available on the actions taken to prevent stones in Germany. A questionnaire that explicitly tackles this area of stone disease does not exist.

We draw three conclusions from the survey: first, urologists must take responsibility for not only treating but also preventing recurrent stone disease. To achieve a balanced responsibility between inpatient and outpatient clinics, adequate reimbursement is essential. Second, urologic departments should extend their general advice on dietary considerations. Additional nutritional counselling by dieticians would fill a gap in urologists' knowledge and lack of time. And third, we need to improve the identification of patients at high risk for recurrence and apply diagnostic algorithms in those patients.

Supplementary Information The online version contains supplementary material available at https://doi.org/10.1007/s00345-021-03813-3.

Author contributions AR: project development, data analysis, and manuscript writing; PR: project development and manuscript editing; $\mathrm{RH}$ : project development and manuscript editing; JN: manuscript editing; PB: project development and manuscript editing. 
Funding Open Access funding enabled and organized by Projekt DEAL. Funded by the Deutsche Forschungsgemeinschaft (DFG, German Research Foundation) - RE 4611/2-1

\section{Declarations}

Conflict of interest The authors declare that they have no conflict of interest.

Ethical standards All procedures performed in studies involving human participants were in accordance with the ethical standards of the institutional and/or national research committee and with the 1964 Helsinki declaration and its later amendments or comparable ethical standards. Ethics approval was not required.

Open Access This article is licensed under a Creative Commons Attribution 4.0 International License, which permits use, sharing, adaptation, distribution and reproduction in any medium or format, as long as you give appropriate credit to the original author(s) and the source, provide a link to the Creative Commons licence, and indicate if changes were made. The images or other third party material in this article are included in the article's Creative Commons licence, unless indicated otherwise in a credit line to the material. If material is not included in the article's Creative Commons licence and your intended use is not permitted by statutory regulation or exceeds the permitted use, you will need to obtain permission directly from the copyright holder. To view a copy of this licence, visit http://creativecommons.org/licenses/by/4.0/.

\section{References}

1. Alelign T, Petros B (2018) Kidney stone disease: an update on current concepts. Adv Urol 2018:3068365. https://doi.org/10. $1155 / 2018 / 3068365$

2. Scales CD, Smith AC, Hanley JM et al (2012) Prevalence of kidney stones in the United States. Eur Urol 62:160-165. https://doi. org/10.1016/j.eururo.2012.03.052

3. Brikowski TH, Lotan Y, Pearle MS (2008) Climate-related increase in the prevalence of urolithiasis in the United States. Proc Natl Acad Sci U S A 105:9841-9846. https://doi.org/10.1073/ pnas.0709652105

4. Daudon M, Jungers P, Bazin D et al (2018) Recurrence rates of urinary calculi according to stone composition and morphology. Urolithiasis 46:459-470. https://doi.org/10.1007/ s00240-018-1043-0

5. Hesse A, Brändle E, Wilbert D et al (2003) Study on the prevalence and incidence of urolithiasis in Germany comparing the years 1979 vs. 2000. Eur Urol 44:709-713. https://doi.org/10. 1016/s0302-2838(03)00415-9

6. Nolde A, Hesse A, Scharrel O et al (1993) Modelprogramm zur nachsorge bei rezidivierenden harnsteinpatienten. Urologe 33(3):148-154
7. Arbeitskreis Harnsteine der Akademie der Deutschen Urologen (2018) S2k-Leitlinie zur Diagnostik, Therapie und Metaphylaxe der Urolithiasis

8. Krepinsky J, Ingram AJ, Churchill DN (2000) Metabolic investigation of recurrent nephrolithiasis: compliance with recommendations. Urology 56:915-920. https://doi.org/10.1016/s00904295(00)00822-0

9. Strohmaier WL (2006) Okonomische aspekte der evidenzbasierten harnsteinmetaphylaxe (Economic aspects of evidence-based metaphylaxis). Urologe A 45:1406-1409. https://doi.org/10.1007/ s00120-006-1220-6

10. Zhe M, Hang Z (2017) Nephrolithiasis as a risk factor of chronic kidney disease: a meta-analysis of cohort studies with 4,770,691 participants. Urolithiasis 45:441-448. https://doi.org/10.1007/ s00240-016-0938-x

11. Shang W, Li Y, Ren Y et al (2017) Nephrolithiasis and risk of hypertension: a meta-analysis of observational studies. BMC Nephrol 18:344. https://doi.org/10.1186/s12882-017-0762-8

12. Siener R, Laube N, Strohmaier WL (2011) Rezidivprävention der Urolithiasis unter Berücksichtigung ökonomischer Aspekte (Recurrence prevention of urolithiasis with respect to economic aspects). Urologe A 50(1276):1278-1282. https://doi.org/10.1007/ s00120-011-2619-2

13. Patel SR, Penniston KL, Nakada SY (2011) Trends in the medical management of urolithiasis: a comparison of general urologists and endourology specialists. Urology 77:291-294. https://doi.org/ 10.1016/j.urology.2010.06.045

14. Türk C, Neisius A, Petř́k A (2021) EAU-Guidelines on urolithiasis 2021. http://uroweb.org/guideline/urolithiasis/

15. Pearle MS, Goldfarb DS, Assimos DG et al. (2016) Medical management of kidney stones: AUA guideline, 192

16. Milose JC, Kaufman SR, Hollenbeck BK et al (2014) Prevalence of 24-hour urine collection in high risk stone formers. J Urol 191:376-380. https://doi.org/10.1016/j.juro.2013.08.080

17. Straub M (2011) Metaphylaxe von harnsteinerkrankungen (Recurrence prevention of kidney stone disease). Urologe A 50:13231332. https://doi.org/10.1007/s00120-011-2640-5

18. Wertheim ML, Nakada SY, Penniston KL (2014) Current practice patterns of urologists providing nutrition recommendations to patients with kidney stones. J Endourol 28:1127-1131. https:// doi.org/10.1089/end.2014.0164

19. Bell Parks R, Penniston K, Nakada SY (2014) Dietitians in practice: utilization of medical nutrition therapy for prevention of kidney stones. Renal Nut Forum 33:8-11

20. Bensalah K, Tuncel A, Raman JD et al (2009) How physician and patient perceptions differ regarding medical management of stone disease. J Urol 182:998-1004. https://doi.org/10.1016/j.juro.2009. 05.025

Publisher's Note Springer Nature remains neutral with regard to jurisdictional claims in published maps and institutional affiliations. 